\title{
Applicability of Capacitive Micromachined Ultrasonic Transducers for the detection of proton-induced thermoacoustic waves
}

Julie Lascaud, Ronaldo Kalunga, Sebastian Lehrack, Hans-Peter Wieser,

Franz Siegfried Englbrecht, Matthias Würl, Walter Assmann,

Alessandro Stuart Savoia and Katia Parodi

(C) 2019 IEEE. Personal use of this material is permitted. Permission from IEEE must be obtained for all other uses, in any current or future media, including reprinting/republishing this material for advertising or promotional purposes, creating new collective works, for resale or redistribution to servers or lists, or reuse of any copyrighted component of this work in other works.

Cite as: Lascaud, J., Kalunga, R., Lehrack, S., Wieser, H. P., Englbrecht, F. S., Würl, M., Assman, W., Savoia, A.S. \& Parodi, K., Applicability of Capacitive Micromachined Ultrasonic Transducers for the detection of proton-induced thermoacoustic waves. 2019 IEEE International Ultrasonics Symposium (IUS) (pp. 143-146). IEEE (2019, October).

DOI: $10.1109 /$ ULTSYM.2019.8926023 


\title{
Applicability of Capacitive Micromachined Ultrasonic Transducers for the detection of proton-induced thermoacoustic waves
}

\author{
Julie Lascaud*, Ronaldo Kalunga*, Sebastian Lehrack*, Hans-Peter Wieser*, Franz Siegfried Englbrecht*, \\ Matthias Würl*, Walter Assmann*, Alessandro Stuart Savoia ${ }^{\dagger \ddagger}$ and Katia Parodi*‡ \\ $\ddagger$ Both authors contributed equally \\ *Ludwig-Maximilians-Universität München, Garching b. Munich, Germany \\ Email: katia.parodi@physik.uni-muenchen.de \\ ${ }^{\dagger}$ Department of Engineering, Roma Tre University, Rome, Italy \\ Email: alessandro.savoia@uniroma3.it
}

\begin{abstract}
This study investigates the application of broadband capacitive micromachined ultrasonic transducers (CMUT) to ionoacoustics (i.e., the thermoacoustic emissions induced by the energy deposition of ion beam) over a wide frequency range from hundreds of $\mathrm{kHz}$ to a few MHz. A water tank was irradiated by a $20 \mathrm{MeV}$ pulsed proton beam. The frequency and amplitude of the ionoacoustic waves were modulated by adding material before to penetrate into the water tank to change the beam energy and its spatial dimensions. The measurements were performed with a $12 \mathrm{MHz}$ CMUT prototype and compared to ones obtained from a commercial 3.5 MHz piezoeletric transducer as well as to in silico studies employing the $k$-Wave Matlab toolbox in combination with FLUKA Monte Carlo simulations to derive the dose (i.e., energy deposition per mass) and initial pressure distribution. Comparison of the experimental and in silico results show that the CMUT bandwidth is wide enough to measure the signal without any degradation or attenuation of the frequency content in the investigated frequency range, thus ensuring accurate reconstruction of the dose distribution and potential bi-modality system for the co-registration of ionoacoustic and ultrasound imaging.
\end{abstract}

Index Terms-CMUT, ionoacoustics, proton range verification

\section{INTRODUCTION}

Compared to other external radiation-based cancer treatments, ion beam therapy allows a local irradiation of cancerous tumors, with better sparing of the surrounding healthy tissues and consequently reduced the treatment side effects. Indeed, due to the characteristic inverse depth dose profile of heavy charged particles, the dose (i.e., amount of energy deposited per irradiated mass) is mainly confined at the end of beam range, so-called Bragg peak. However, beam delivery and patient positional/anatomical uncertainties may cause ions to stop at different locations than planned, thereby compromising the treatment quality in daily clinical practice. A precise in vivo localization of the Bragg peak is thus essential to fully exploit the benefits offered by ion therapy. To this end, ionoacoustics is an emerging modality, which is based on the detection of the pressure waves generated by the ions stopping at the Bragg peak. The sharp energy deposition induced by narrow and pulsed ion beams leads to a local temperature increase and a subsequent thermoacoustic emission [1]. The wave characteristics depend on the spatial heating function, correlated to the dose distribution, whereby the Bragg peak position can be determined by a time-of-flight measurement on beam axis, whereas the dose can be reconstructed from multiple point measurements [2]. The relative simplicity of this modality and the possibility to co-registrate anatomical ultrasound imaging make ionoacoustics a strong candidate for real-time in vivo dose monitoring for accessible anatomical locations (e.g., prostate, breast, liver). However, the main challenge is to detect the pressure waves which are, for biomedical applications, characterized by a very low-pressure $(\leq 1-100 \mathrm{mPa})$ and lowfrequency $(\leq 10-100 \mathrm{kHz})$. In addition, the sensor must have a sufficiently large bandwidth to not distort the ionoacoustic signal allowing an accurate dose reconstruction. Ultimately, the same very broadband transducer could be used for ionoacoustic and ultrasound imaging co-registration. As previously demonstrated [3], capacitive micromachined ultrasonic transducers (CMUT) have a larger bandwidth and higher sensitivity compared to conventional PZT-based transducers, which was shown to significantly improve the quality of photoacoustic images. This study investigates for the first time the application of CMUT to ionoacoustics over a wide frequency range from hundreds of $\mathrm{kHz}$ to few $\mathrm{MHz}$.

\section{Methodology}

\section{A. Experimental setup}

The experimental campaign was performed at the Tandem accelerator of the Maier-Leibnitz-Laboratory (Munich, Germany), using a $20 \mathrm{MeV}$ proton beam pulsed by a chopper/buncher system and resulting in a rectangular-shaped proton pulse [4]. The proton pulse duration was fixed to $100 \mathrm{~ns}$ with an injected current of $2.7 \mathrm{nA}\left(1.72 \times 10^{6}\right.$ protons/pulse $)$. Experiments were carried out in water. The proton beam was penetrating into the water tank through an air channel 
a - Without ripple filter

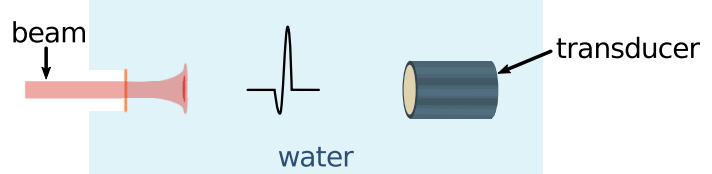

b - With ripple filter

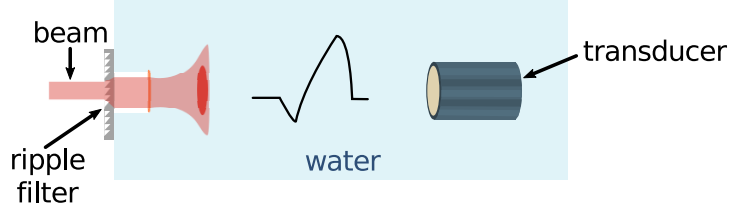

Fig. 1. Schematic representation of the experimental setup a) without the ripple filter to not degrade the proton beam in order to generate $\mathrm{MHz}$ ionoacoustic signals and b) with the aluminium ripple filter to spread out the Bragg peak volume in order to produce a lower ionoacoustic frequency range.

terminated by a $50 \mu \mathrm{m}$-thick polyimide film and the transducer was placed on the beam axis, as represented in Fig.1. The frequency and amplitude of the ionoacoustic waves were modulated by adding a saw-tooth ripple filter in aluminium at the beam entrance. The irregular structure of the ripple filter causes protons to stop at different positions on the beam axis and the higher proton scattering than in water widens the beam lateral dimension. As a consequence, when using the ripple filter the Bragg peak volume is spread in all dimensions, resulting in an ionoacoustic signal at a lower frequency and with a reduced amplitude. A $3.5 \mathrm{~mm}$-diameter single-element reverse-fabricated CMUT probe, designed for two-way operation around $12 \mathrm{MHz}$ [5] and equipped with a low-noise amplifier (LNA) for receive (RX) performance optimization, was used for the measurements. The in-probe LNA, consisting in a high input impedance, high-voltage protected, low-noise voltage buffer (MAX4200, Maxim Integrated, Sunnyvale, CA), was designed for a $-3 \mathrm{~dB}$ RX frequency response in the $40 \mathrm{kHz}$ to $15 \mathrm{MHz}$ range. The pressure measured with the CMUT was compared to measurements performed with a commercial 3.5 $\mathrm{MHz}$ PZT focused transducer (12 mm-diameter, focal length of 1 inch and $80 \%$ bandwidth - V382, Olympus). The measured signals were amplified by $+60 \mathrm{~dB}$ using LNA (HVA10M-60-B, Femto) and acquired through a digital oscilloscope (6404D, picoscope) at a sampling frequency of $312.5 \mathrm{MHz}$.

\section{B. Ionoacoustic simulations}

Experimental results were compared to in silico studies. The proton dose distributions $D$ were computed in 3D by FLUKA Monte Carlo simulations and converted into an initial pressure $p_{0}$ distribution using (1), where $\Gamma$ is the Grüneisen parameter $\left(\Gamma=0.11\right.$ in water at $20{ }^{\circ} \mathrm{C}$ ) and $\rho$ is the medium density $\left(\rho_{\text {water }}=998 \mathrm{~kg} / \mathrm{m}^{3}\right.$ at $\left.20^{\circ} \mathrm{C}\right)$.

$$
p_{0}(x, y, z)=\Gamma \times D(x, y, z) \times \rho
$$

Propagation of the ionoacoustic waves was simulated using the k-Wave Matlab toolbox [6], which is based on a k-space pseudo-spectral time-domain model. The pressure distributions were deduced from the FLUKA doses and were used as input to define the amplitude and geometry of the acoustic source, considering an initial problem value (i.e., assuming an ideal delta-spike function as excitation in time-domain). The medium properties were chosen to correspond with a $20{ }^{\circ} \mathrm{C}$ water medium, as in the experiments. The attenuation in water was neglected and perfectly matched layers were implemented to avoid any reflection at the grid boundaries. The evolution of the pressure over time was recorded using an ideal point sensor placed on the beam axis.

\section{RESULTS AND DISCUSSION}

\section{A. Comparison between CMUT and PZT transducers}

The ionoacoustic signals measured for the $20 \mathrm{MeV}$ proton beam without the ripple filter are shown in Fig.2 for the two transducers. They are compared to the simulation for an ideal point detector in time- and frequency-domain. In the timedomain, the signal is composed of two pulses. The first pulse is produced at the Bragg peak position (direct signal) and is the result of the temperature gradient induced by the energy deposition of the proton beam in water. For short proton pulse durations (i.e., bellow the stress-confinement limit, in this case $\leq 200 \mathrm{~ns}$ ), the pressure wave is the first spatial derivative of the heating function (i.e., the dose distribution). Therefore, the direct signal is typically a bipolar signal, composed of a positive part subsequent to the temperature increase followed by a negative pressure of lower amplitude, as can be seen from the simulated signal in Fig.2. The second pulse is created at the air/window/water interface (window signal). It is the result of both the discontinuity of the dose (i.e., protons are not stopped in the same way depending on the material) and the variation of the Grüneisen parameter (i.e., different conversion from energy to pressure depending on the material). The resulting temperature gradient leads to a negative pulse as observed in Fig.2. In contrast to the PZT transducer, the time signal measured with the CMUT agrees well with the expected signal obtained from the simulation. The bipolar shape of the direct signal as well as the negative window signal are both clearly observable. Differently, the signal measured with the PZT detector is distorted, the direct signal starts with a negative pulse and the window signal is bipolar. This distortion is due to the limited bandwidth of the PZT transducer. Indeed, as can be seen in the spectra, the PZT transducer mainly detects the frequencies around $3.5 \mathrm{MHz}$ (center frequency of the PZT transducer) and attenuates the lower frequencies. In comparison, the large bandwidth of the CMUT allows to detect the global frequency content of the signal and consequently to preserve the shape of the time signal, fundamental for an accurate reconstruction of the dose.

\section{B. Low frequency detection with the CMUT}

Fig.3 shows the ionoacoustic signal obtained with the CMUT when adding the ripple filter in front of the air entrance 
a) lonoacoustic signals in time-domain

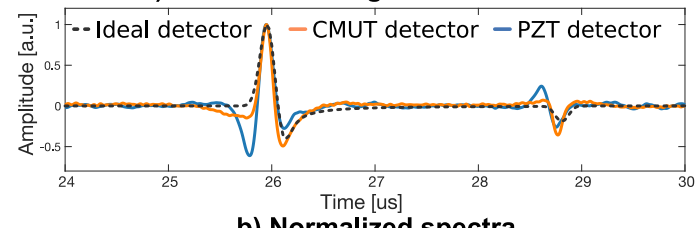

b) Normalized spectra

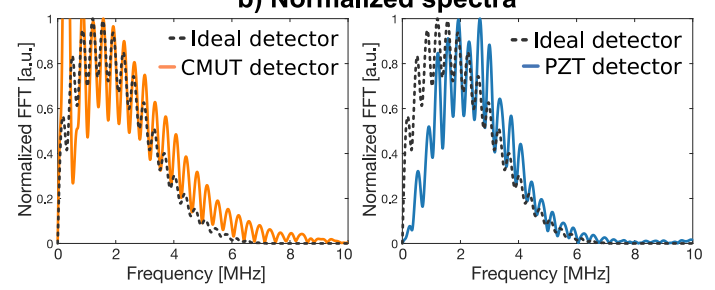

Fig. 2. Ionoacoustic signals without ripple filter a) in time-domain measured with the CMUT (orange) and with the PZT transducer (blue) compared to the simulation (black) and b) normalized FFT.

channel of the water tank. The ripple filter increases the axial dimension of the Bragg peak region from $300 \mu \mathrm{m}$ to $3 \mathrm{~mm}$ and consequently reduces the ionoacoustic frequency by a factor 10. Even in this low frequency range $(70 \mathrm{kHz}$ to $400 \mathrm{kHz}$ at $-6 \mathrm{~dB}$ ) the CMUT successfully measured the ionoacoustic signal without any degradation, as demonstrated in Fig.3. It should be noted that, in this frequency range no measurement with the PZT transducer was possible. These findings suggest that CMUT offer a promising technology for the development of ionoacoustic detectors, for which the CMUT broadband capability ensures preservation of the complete frequency content of the ionoacoustic signal, which is favorable for an accurate reconstruction of the dose distribution. Moreover, in clinical practice the proton beam energy is changed during patient treatment to vary the proton beam range such that the tumor is fully cover in depth. As the energy is increased, the axial dimension of the Bragg peak is widened, leading to variations of the ionoacoustic frequency from hundreds of $\mathrm{kHz}$ down to tens $\mathrm{kHz}$. Therefore, a sufficiently broadband transducer, as CMUT, allows the use of the same sensor all along the treatment while in contrast narrow bandwidth transducer would require changing the detector at different treatment steps.

\section{CONCLUSiON}

In this study we investigated for the first time the applicability of a CMUT prototype for the detection of ionoacoustic pressure waves over a wide frequency range, from hundreds of $\mathrm{kHz}$ to a few $\mathrm{MHz}$. Ionoacoustic measurements were firstly performed for a $20 \mathrm{MeV}$ mono-energetic proton beam in water, resulting in ionoacoustic frequencies in the $\mathrm{MHz}$ range. The signal measured with a $12 \mathrm{MHz}$ CMUT prototype was compared to the one obtained from a commercial 3.5 $\mathrm{MHz}$ focused PZT transducer. It was shown that the larger bandwidth of the CMUT allowed acquisition of the ionoacoustic signal without any distortion or attenuation of the frequency content compared to the measurements with the a) lonoacoustic signals in time-domain

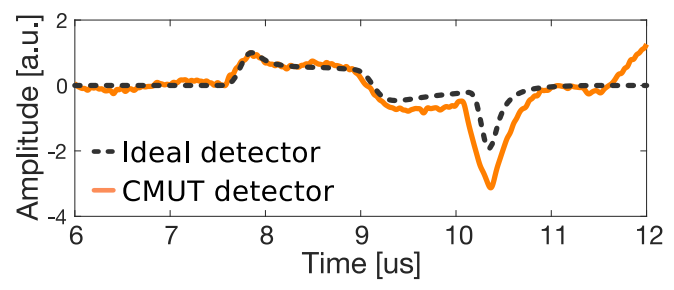

b) Normalized spectra

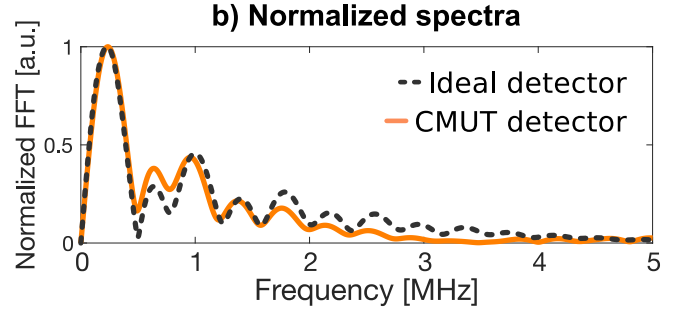

Fig. 3. Ionoacoustic signal measured with the CMUT (orange) for the $20 \mathrm{MeV}$ proton beam degraded with the aluminium ripple filter compared to simulation for an ideal point detector (black) a) in time-domain and b) normalized FFT of the signals.

PZT transducer, when compared to the ideal signal predicted by the in silico study. Thereafter, the $20 \mathrm{MeV}$ proton beam was degraded using an aluminium ripple filter in order to spread out the Bragg peak region and consequently reduce the ionoacoustic frequency content to the hundreds of $\mathrm{kHz}$ range which is outside the detectability range of the employed PZT transducer. It was shown that the CMUT prototype was able to almost perfectly capture the ionoacoustic signal in this lowfrequency range. In conclusion, the broad bandwidth of the CMUT ensures preservation of the ionoacoustic signal over a wide range of proton beam energies, which is favorable for an accurate reconstruction of the dose distribution in a clinical case scenario. Moreover, the measurements were carried out with a high-frequency transducer, suggesting that CMUT are suitable for the development of bi-modality ultrasound systems, as required for the co-registration of ionoacoustic and ultrasound imaging to overlap the dose distribution with the patient anatomy. Current work is devoted to the application of alternative CMUT receiving circuit architectures, such as transimpedance and charge amplifier topologies [7], to optimize signal to noise ratio thus allowing the detection of lower acoustic pressure levels.

\section{ACKNOWLEDGMENT}

The authors acknowledge financial support from the European Research Council (grant agreement number 725539) as well as the German Research Foundation (project number 403225886 and Excellence Cluster "Munich Center for Advanced Photonics"). The authors are grateful to Barbara Mauti for her support in the packaging of the CMUT probe used in the experiments.

\section{REFERENCES}

[1] K. C. Jones, C. M. Seghal, and S. Avery, "How proton pulse characteristics influence protoacoustic determination of proton-beam range: 
simulation studies," Physics in Medicine \& Biology, vol. 61, no. 6, p. 2213, 2016.

[2] S. Kellnberger, W. Assmann, S. Lehrack, S. Reinhardt, P. Thirolf, D. Queirós, G. Sergiadis, G. Dollinger, K. Parodi, and V. Ntziachristos, "Ionoacoustic tomography of the proton bragg peak in combination with ultrasound and optoacoustic imaging," Scientific reports, vol. 6, p. 29305, 2016.

[3] M. Vallet, F. Varray, J. Boutet, J.-M. Dinten, G. Caliano, A. S. Savoia, and D. Vray, "Quantitative comparison of pzt and cmut probes for photoacoustic imaging: Experimental validation," Photoacoustics, vol. 8, pp. 48-58, 2017.

[4] W. Assmann, S. Kellnberger, S. Reinhardt, S. Lehrack, A. Edlich, P. Thirolf, M. Moser, G. Dollinger, M. Omar, V. Ntziachristos et al., "Ionoacoustic characterization of the proton bragg peak with submillimeter accuracy," Medical physics, vol. 42, no. 2, pp. 567-574, 2015.

[5] G. Caliano, A. S. Savoia, C. Longo, A. Caronti, M. Pappalardo, A. Iula, and S. Rothmann, "cmut sensor for applications as a wide-band acoustic receiver in the $\mathrm{mhz}$ range," in 2010 IEEE International Ultrasonics Symposium. IEEE, 2010, pp. 1869-1872.

[6] B. E. Treeby and B. T. Cox, "k-wave: Matlab toolbox for the simulation and reconstruction of photoacoustic wave fields," Journal of biomedical optics, vol. 15, no. 2, p. 021314, 2010.

[7] M. Sautto, A. S. Savoia, F. Quaglia, G. Caliano, and A. Mazzanti, "A comparative analysis of cmut receiving architectures for the design optimization of integrated transceiver front ends," IEEE transactions on ultrasonics, ferroelectrics, and frequency control, vol. 64, no. 5, pp. 826838, 2017. 